\title{
Application Analysis of Electronic Power Transformer in Photovoltaic Power System
}

\author{
CHEN GuoLiang ${ }^{1, a}$ \\ ${ }^{1}$ Nantong Institute of Technology, Nantong, Jiangsu 226002 China \\ a13962955641@163.com
}

Keywords: Photovoltaic power generation system; Electronic power transformer; Micro-grid; Distribution network;

\begin{abstract}
With the continuous development of economy and population growth, the demand for energy is growing. Due to limited reserves of traditional fossil fuels and the pollution on environment, it is urgent to develop and utilize new energy sources. Under such circumstances, all countries are vigorously developing new energy power generation. As a renewable clean energy, photovoltaic power generation is attracted many countries. The photovoltaic power generation system is accessed to the distribution network through the electronic power transformer. According to the characteristics of the photovoltaic inverter system, the factors affecting the design of the dry-type transformer for photovoltaic power generation are analyzed, which lays the foundation for the follow-up research.
\end{abstract}

\section{Introduction}

The photovoltaic power generation system that is connected to the grid and transmits power to the grid has the advantages including abundant resources, safe and reliable, low dependence on resource distribution and geographical area, high energy quality and short construction period. Because it belongs to green energy projects, the establishment of photovoltaic power plants has certain dependence on construction area and meteorological environment. Therefore it has been widespread concern in the community. As a kind of clean and renewable energy, photovoltaic power generation has drawn more and more attention and development from all over the world. The photovoltaic power generation step-up transformer connects the low-voltage alternating current generated by photovoltaic inverter. Considering the reliability of power plant operation, step-up transformers often use dry-type transformers. Because of certain voltage harmonics and direct current components in front-end inverter circuits, the dry-type step-up transformers for photovoltaic power generation should be specially considered in the design to ensure product reliability

\section{Photovoltaic power generation system analysis}

A Equivalent circuit model of photovoltaic cells

Photovoltaic cell equivalent circuit is shown in Figure 1.

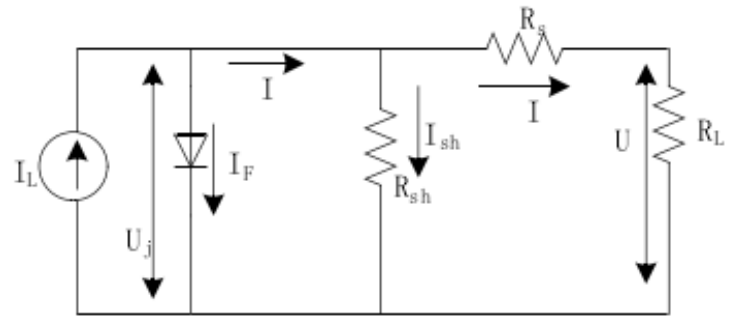

Figure 1 Photovoltaic cell equivalent circuit

It consists of an ideal current source $\mathrm{I}_{\mathrm{L}}$, an antiparallel diode, a series resistor Rs, and a shunt resistor Rsh. Equation 1 is the relationship between the PV cell's output voltage and current. 


$$
I=I_{L}-I_{F}\left[\exp \frac{q}{A k T}\left(U+I R_{s}\right)-1\right]-\frac{U+I R_{s}}{R_{s h}}
$$

Formula 1

Where I is the battery output current, $\mathrm{U}$ is the battery output voltage, $\mathrm{I}_{\mathrm{F}}$ is the reverse saturation current, $\mathrm{T}$ is the thermodynamic temperature of the battery $(\mathrm{K}), \mathrm{k}$ is the Boltzmann constant $\left(1.38 \times 10^{-23} \mathrm{~J} / \mathrm{K}\right)$, A Is the $\mathrm{p}-\mathrm{n}$ junction coefficient of a semiconductor cell in a photovoltaic cell, and $\mathrm{q}$ is the electron charge amount $\left(1.6 \times 10^{-19} \mathrm{C}\right)$. Ideally, Rs can be considered as zero, Rsh can be considered as infinity, then Eq. 1 can be reduced to Eq.

$$
I=I_{L}-I_{F}\left(\exp \frac{q}{A k T} U-1\right) \quad \text { Formula } 2
$$

The I-V curve of the photovoltaic cell can be drawn from the above output current and voltage equations as shown in FIG. 2.

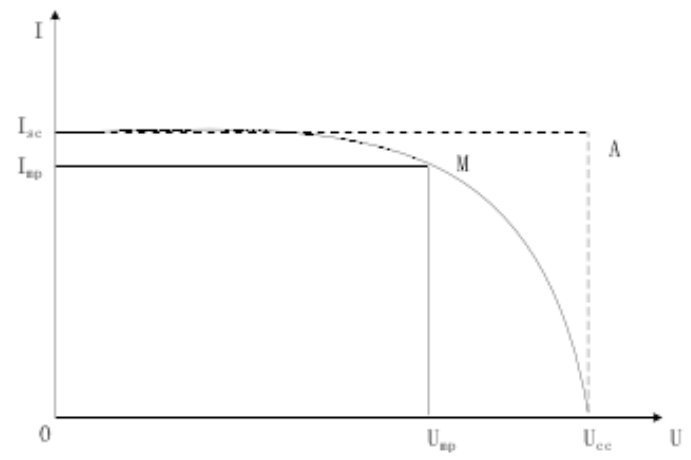

Figure 2 photovoltaic cells I-V characteristic curve

\section{B. Photovoltaic cell output characteristics}

Figure 2 shows the PV cell output I-V curve. It can be seen from equation 1 that the output of photovoltaic cells is related to the intensity and temperature of sunlight. To better understand the output characteristics of photovoltaic cells, two sets of IV curves with different temperatures and the same temperature are plotted in Figure 3 and Figure 4 respectively. According to the graph, you can know the impact of light intensity and temperature on the output voltage and current of PV cells.
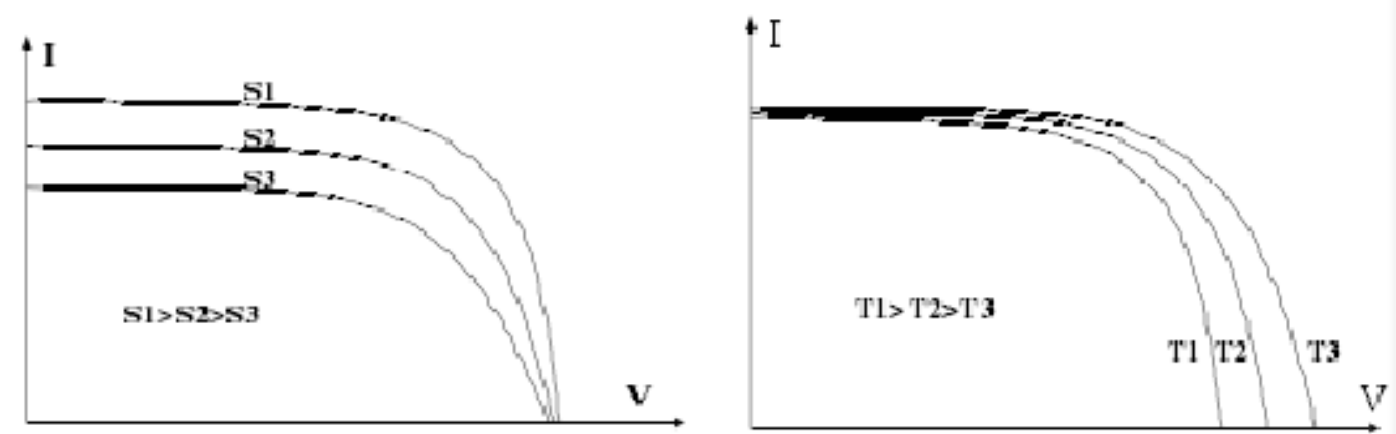

Figure $3 \mathrm{I}-\mathrm{V}$ curve of the same temperature is not the same light Figure $4 \mathrm{I}-\mathrm{V}$ curve of the same temperature is not the same light
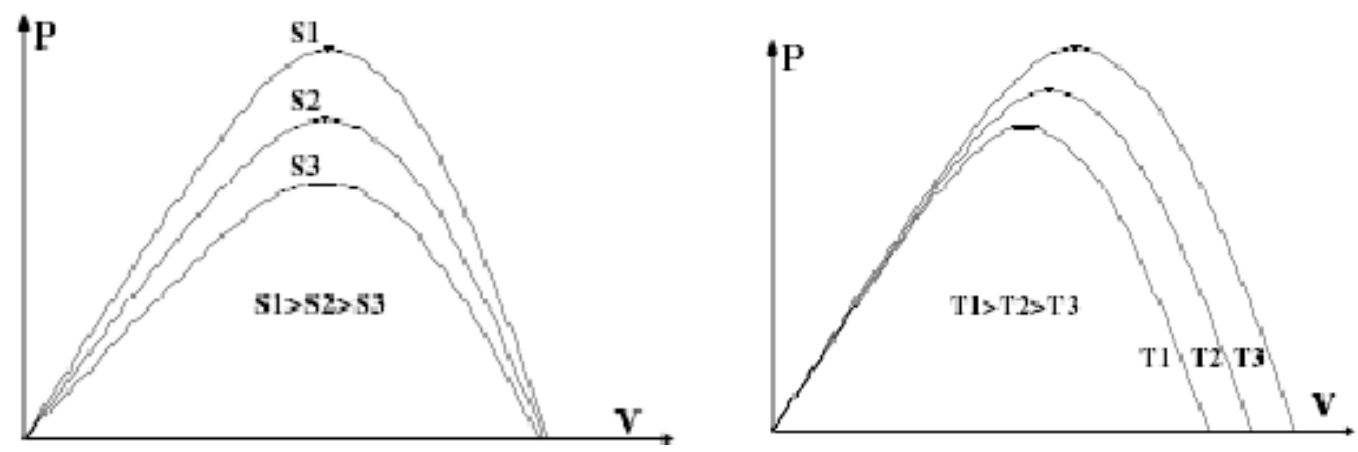
Figure $5 \mathrm{P}-\mathrm{V}$ curves with different temperatures at the same time Figure $6 \mathrm{P}-\mathrm{V}$ curve of the same temperature is not the same light

\section{Influences of DC Bias and Design Considerations}

PV inverters often use PWM inverter circuit and inverter output is PWM pulse. There is no DC component in theory. But in the actual operation, the waveform is usually in asymmetric conditions, mainly for the following reasons: (1) there is a difference in the inverter conduction saturated pressure drop. the storage time is not the same and the output voltage waveform range of positive and negative are not equal; (2) the control circuit output drive pulse positive and negative semi-axis are asymmetry; (3) The control system in the process of dynamic regulation of the modulated wave prone to positive and negative half-weeks area, causing the output voltage imbalance. Therefore, the output circuit in the inverter also had a DC bias, making the input voltage of the transformer in the DC component is difficult to avoid. Without the isolation transformer inverter, the situation is more obvious, according to the standard GB / T 19939-2005 "PV system requirements for grid connection", the DC current component must not exceed 1\% of its AC rating, and usually inverter manufacturers control the value of $0.5 \%$. DC bias existing, it will cause the core of the positive and negative half-cycle flux asymmetry. Assuming that the original design of the maximum magnetic flux density positive and negative semi-cycle were (+ Bs and -Bs), the flux density also changes. the occurrence of DC Bias, the two become $(+\mathrm{Bs}+\mathrm{Bd}$, $-\mathrm{Bs}+\mathrm{Bd})$, magnetic flux density increased half weeks, the excitation current, the noise also increases and system efficiency is reduced. If the DC bias is too large, it will cause core saturation and excitation current distortion. Therefore photovoltaic inverter system need to fully consider the impact of DC bias on the step-up transformer, the use of circuit simulation software to establish single-phase equivalent DC bias model, which is shown in Figure 7:

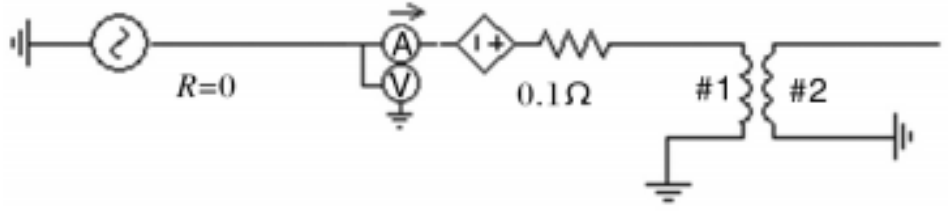

Figure 7 single-phase DC bias equivalent simulation circuit

Simulation results show that there is no DC bias and $0.5 \%$ DC bias, $1 \%$ DC bias case, the transformer excitation current curve shown in Figure 8. It can be seen that the excitation current of the transformer also increases significantly with the increasing of DC bias. Therefore, effective measures must be taken from the design of the transformer to reduce the influence of DC bias on the transformer and inverter, especially in the selection of the flux density of the transformer. At the same time, due to the filtering effect of the step-up transformer on the DC component, the DC component does not flow into the grid and the stability of the overall power plant is improvd.
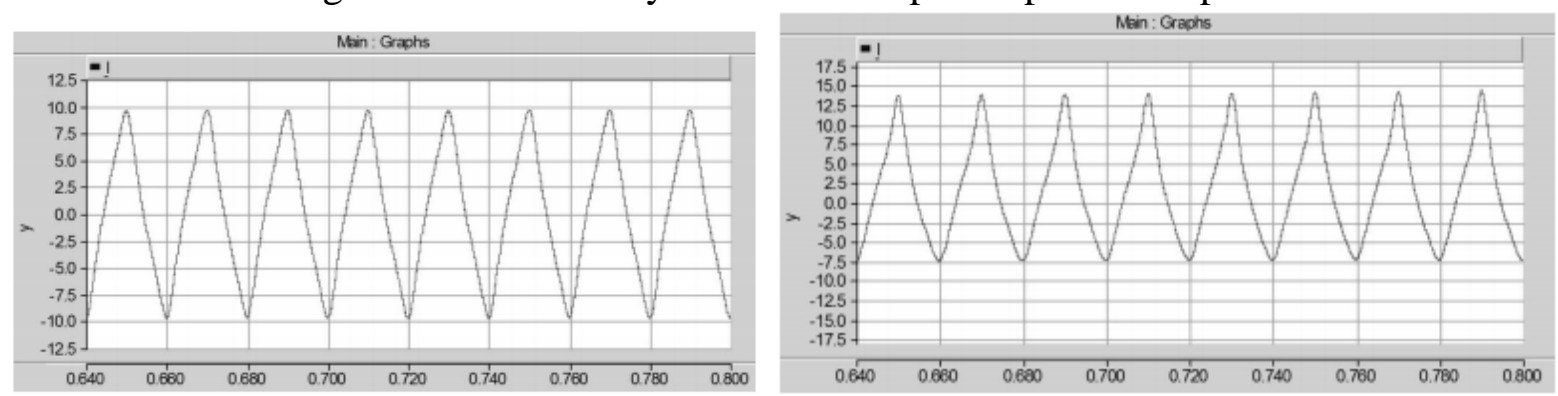

(a) Excitation current simulation results without DC bias (b ) $0.5 \%$ DC component, the excitation current simulation results 


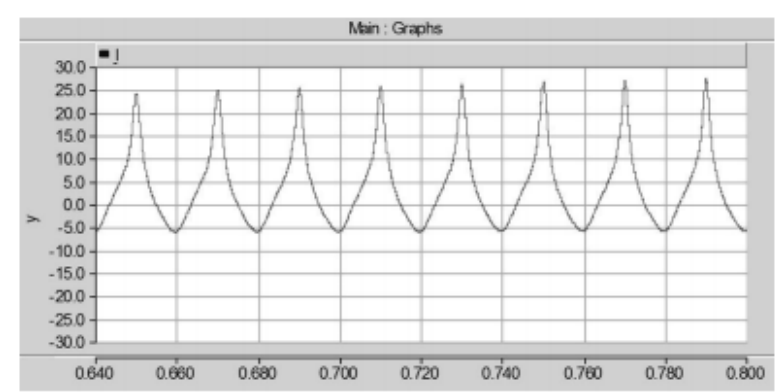

(c) DC component $1 \%$, the excitation current simulation results

Fig.8 Excitation current simulation results under different DC components

\section{Application of Electronic Power Transformer in Photovoltaic Power System}

In the microgrid, the voltage level is lower and the system capacity is smaller. The fluctuation of the power supply in the system has a greater impact on the system. According to the output characteristics of the microgrid and the photovoltaic system, when the photovoltaic system is connected to the microgrid through the electronic power transformer, the topology of the electronic power transformer is selected. Since the output of the photovoltaic system is DC, the electronic power transformer connects the DC system and the AC system, and the operation modes on both sides of the system may not be kept in synchronization. It is possible to use a hybrid electronic power transformer topology. Figure 9 shows the topology of an electronic power transformer applied to a photovoltaic access microgrid.

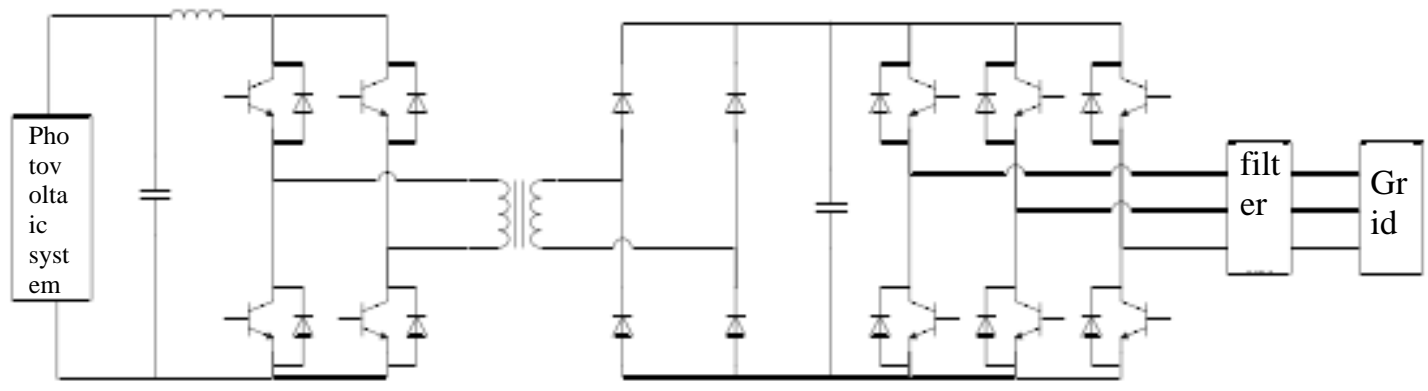

Fig.9 Photovoltaic access microgrid power transformers topology

In this structure, the primary single-phase full-bridge inverter can chop DC and modulate DC into high-frequency square wave. The diode rectifier changed into the DC after the high-frequency transformer coupled to the secondary side. It changed into the desired AC and then inverted the grid after three-phase inverter circuits. Throughout the work process, the primary and secondary do not need to be synchronized. The primary side of the DC used voltage control mode and the secondary side of the constant power or constant voltage used control mode. At the same time, it increases the damping of the whole system and improves the system stability. The grid-connected side of the PV system under operation runs the unit power factor, and the grid-side voltage, current and power waveforms are as follows:

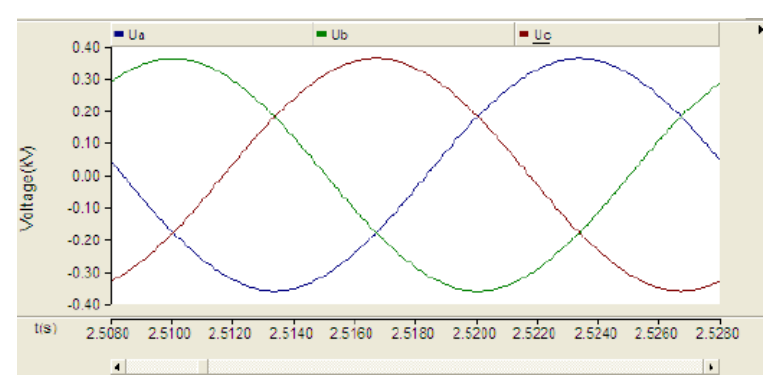

Figure 10 and the grid-side three-phase voltage waveform

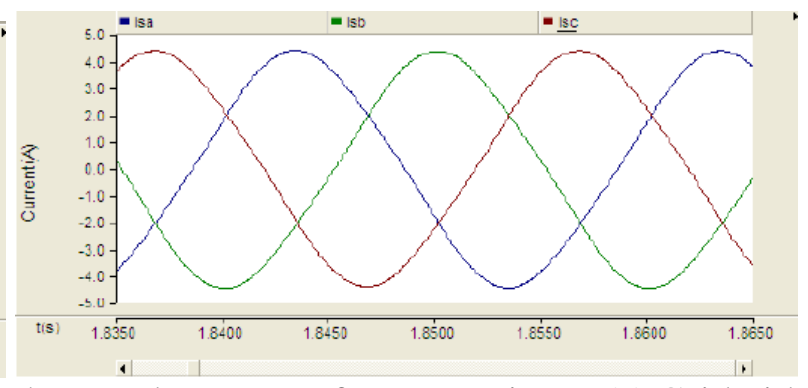

Figure 11 Grid-side three-phase current waveform

After the photovoltaic power generation system is integrated into the microgrid using an electronic power transformer, the voltage and current are all standard sinusoidal waveforms. Photovoltaic 
power generation systems incorporating microgrids through electronic power transformers have some accidental operational capabilities and are capable of delivering power to the microgrid in the event of an accident maintaining the stability of the port voltage and current.

\section{Conclusion}

Electronic power transformers have excellent characteristics and can solve many problems of modern power systems. The paper studies the photovoltaic power generation system through the access of electronic power transformers to the power grid, mainly studies the basic output characteristics of photovoltaic systems and the grid-connected the transient and steady-state characteristics.

\section{Acknowledgements}

Nantong science and technology project project, project name: Research on micro grid control strategy based on distributed wind power generation system. Project number: GY12016044

\section{References}

[1] Jian Yuan, Xiqiang Chang, Wei Wei, Xinjia Niu, Chen Wang. Influence of Large Scale Photovoltaic Power Generation on Relay Protection in Xinjiang Power Grid [J]. Electrical Technology,2015,10:27-33.

[2]Dan Lv, JiaLong Pan. Calculation and Selection of Grounding Transformer and Arc Suppression Coil in Photovoltaic System [J]. Renewable Energy,2013,09:18-20+25.

[3]Shengzhong Zhang. Analysis of Photovoltaic Power Generation Efficiency under Different Access Systems [J]. Renewable Energy,2014,01:29-33.

[4]Chao Zheng, Junjie Lin, Jian Zhao, Canhui Sheng, Feng Gao. Transient Power Characteristics and Voltage Control of Large Scale PV Grid Connection System [J]. Chinese Society for Electrical Engineering,2015,05:1059-1071.

[5]Lin Zhou, Wei Ren, Bo Liao, Yang Cao, Nianbing Shao, Xiao Du. Grid-connected photovoltaic power station reactive voltage control [J]. Transactions of China Electrotechnical Society,2015,20:168-175. 